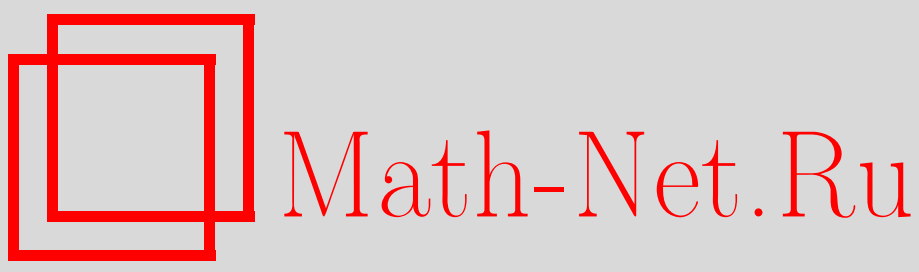

W. Chelkh, I. Ly, N. N. Tarkhanov, A remark on the laplace transform, Sibirsk. Mat. Zh., 2020, Volume 61, Number 4, 946-955

DOI: https://doi.org/10.33048/smzh.2020.61.415

Use of the all-Russian mathematical portal Math-Net.Ru implies that you have read and agreed to these terms of use http://www . mathnet.ru/eng/agreement

Download details:

IP : 3.91 .87 .62

April 26, 2023, 06:42:23

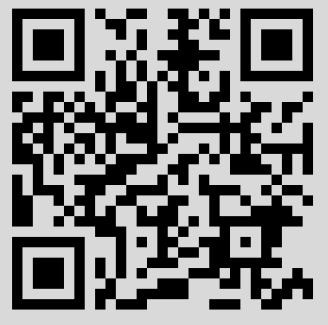


Сибирский математический журнал Июль-август, 2020. Том 61, № 4

УДК 517.955

\title{
ЗАМЕЧАНИЕ О ПРЕОБРАЗОВАНИИ ЛАПЛАСА
}

\section{В. Челк, И. Ли, Н. Н. Тарханов}

\begin{abstract}
Аннотация. При изучении задачи Коши для решения уравнения теплопроводности в цилиндрической области с начальными данными на боковой поверхности с помощью метода Фурье возникает проблема вычисления обратного преобразования Лапласа целой функции $\cos \sqrt{z}$. В рамках стандартной теории преобразования Лапласа эта проблема не имеет решения. Приведена явная формула для обратного преобразования Лапласа для $\cos \sqrt{z}$ с использованием теории аналитических функционалов. Это решение хорошо подходит для того, чтобы эффективно развить регуляризацию решения задачи Коши для параболических уравнений с данными на нехарактеристических поверхностях.
\end{abstract}

DOI 10.33048/smzh.2020.61.415

Ключевые слова: преобразование Фурье - Лапласа, распределение с односторонним носителем, голоморфная функция, аналитический функционал.

\section{1. Введение}

Преобразование Фурье - один из наиболее важных методов в изучении многих задач математического анализа и в особенности дифференциальных уравнений. Применимость этого метода ограничена различными предположениями относительно поведения функций на бесконечности. В классических работах ими служат условия типа интегрируемости функции или ее степеней. В работах физического содержания преобразование Фурье применяется, часто без достаточных объяснений, к функциям, возрастающим не быстрее некоторой степени выражения $\langle t\rangle=\sqrt{1+|t|^{2}}$. Строгая теория преобразований Фурье медленно растущих функций разработана в [1] и далее развита в [2] с помощью другого метода. Шварц в своей книге [3] интерпретировал преобразование Фурье как функционал и разработал систематическую теорию специально для этого класса функций.

Таким образом, в указанных работах не хватало преобразования Фурье для быстро растущих функций таких, как $\exp t, \exp t^{2}$ и т. д. С другой стороны, распространение преобразования Фурье на быстро растущие функции очень важно. Это видно на примере применения метода Фурье к традиционной задаче, а именно задаче Коши для решения уравнений в частных производных. Отметим работу [4], в которой изучается корректность задачи Коши с ограниченными начальными данными. Последнее условие возникает не в силу природы характера самой задачи, а как результат использования метода Фурье. Известно, например, что существование и единственность решения задачи Коши для гиперболических уравнений имеет место без ограничений на рост исходных функций на бесконечности, поскольку значения, принимаемые решением в произвольной точке, зависят только от значений этих функций внутри характеристического конуса. Если $u \in L^{2}(\mathbb{R})$ имеет носитель на промежутке $[a, b]$, то

(с) 2020 Челк В., Ли И., Тарханов Н. Н. 
ее преобразование Фурье определяет целую функцию экспоненциального типа, a ее рост в нижней полуплоскости определяется наибольшим возможным $a$.

В точности обратное к изложенному выше наблюдению дает теорема Пэли - Винера в [5], далее обобщенная в [6] несколькими способами, использующими, например, распределения в $\mathbb{R}^{n}$ с носителем на компакте или в полупространстве (см. теоремы 7.3.1, 7.4.2 и 7.4.3 в [7]). Стоит упомянуть, например, статьи [8-12] - одни из многих, содержащих различные версии теоремы Пэли Винера.

Будем рассматривать только случай размерности $n=1$. Теорема вышеописанного типа имеет следующий вид: голоморфная функция $f$ в нижней полуплоскости может быть представлена как преобразование Фурье распределения с носителем на $[a, \infty)$ для некоторого $a \in \mathbb{R}$, если рост $f$ удовлетворяет определенным оценкам. Особенным условием является требование к $f$ быть экспоненциального типа по крайней мере на полуплоскости $\operatorname{Im} z<y_{0}$ для некоторого $y_{0}<0$, зависящего от $a$. В [8] преобразование Фурье строится для функций, возрастающих неограниченно быстро на бесконечности. Отличительной чертой данной работы является выход в комплексную плоскость и рассмотрение функционалов на различных классах (обычно целых) функций. При этом подходе теоремы единственности базируются на рассуждениях, основанных на теореме Фрагмена - Линделефа из теории целых функций. Дальнейшее развитие теории см. в [13-15] и т. д.

В [16] метод Фурье применен для построения явного приближенного решения задачи Коши для решения уравнения теплопроводности в цилиндре с начальными данными на части боковой поверхности. Поскольку за исключением одного фрагмента формула содержит обратное преобразование Лапласа целой функции $\cos \sqrt{\tau}$ комплексной переменной $\tau=t+\imath s$ из $\mathbb{C}$, она не может быть вычислена в рамках классического операционного анализа. По этой причине в [16] есть обращение к более ранней работе [8]. Целью данной статьи является завершение конструкции из [16] с помощью детального применения методики из [8] и явного нахождения обратного преобразования Лапласа для $\cos \sqrt{\tau}$. Данный подход применим для большого класса задач Коши при решении как параболических уравнений, так и параболических уравнений с обратным временем в цилиндре над ограниченной областью $\mathscr{X}$ в $\mathbb{R}^{n}$ с начальными данными в полосе на боковой поверхности цилиндра.

\section{2. Нехарактеристическая задача Коши}

Данная статья мотивирована работой [16], в которой развивается подход к изучению динамики решения эллиптического уравнения в ограниченной области $\mathscr{X} \subset \mathbb{R}^{n}$ с данными задачи Коши, заданными на открытом участке $S$ граничной поверхности $\partial \mathscr{X}$. А именно, пусть $A$ - эллиптический оператор в окрестности замыкания $\mathscr{X}$. Рассмотрим задачу Коши

$$
u_{t}^{\prime}=A u+f \text { в } \mathscr{C}, \quad t(u)=u_{0} \text { на } S \times(0, \infty)
$$

для уравнения эволюции в полуцилиндре $\mathscr{C}=\mathscr{X} \times(0, \infty)$, где $t(u)-$ данные Коши для неизвестной функции $u$ в $\mathscr{C}$ относительно оператора $A$, а $f$ и $u_{0}$ функции в $\mathscr{C}$ и $S \times(0, \infty)$ соответственно. Полоса $S \times(0, \infty)$, очевидно, является нехарактеристической частью границы $\mathscr{C}$ относительно оператора эволюции $\partial_{t}-A$, а значит, $(2.1)$ - нехарактеристическая задача Коши. 
Применяя преобразования Лапласа

$$
\hat{u}(x, \tau):=\mathscr{L} u(x, \tau)=\int_{0}^{\infty} e^{-\imath \tau t} u(x, t) d t
$$

ко всем уравнениям задачи $(2.1)$ при $t \in(0, \infty)$, получаем семейство задач Коши

$$
(A-\imath \tau) \hat{u}(\cdot, \tau)=-u(\cdot, 0)-\hat{f}(\cdot, \tau) \text { в } \mathscr{X}, \quad t(\hat{u})(\cdot, \tau)=\hat{u}_{0}(\cdot, \tau) \text { на } S
$$

в области $\mathscr{X}$, параметризованной комплексным параметром $\tau$, пробегающим вдоль горизонтальной линии в нижней полуплоскости. Для произвольного фиксированного $\tau$ легко видеть, что (2.2) - задача Коши для эллиптического оператора $A-\imath \tau$ на $\mathscr{X}$ с данными на части границы $S$. Известно, что если $S$ не совпадает со всей границей, то эта задача нестабильна в естественной постановке. Это свидетельствует о некорректности задачи (2.1) (см. [17]).

Задача Коши (2.2) решается методом, разработанным в [17]. Существенным инструментом в изучении некорректных задач является понятие функции Карлемана. В отличие от функции Грина для эллиптической граничной задачи, функция Карлемана порождает приближенное решение задачи (ср. с формулой (7.1) ниже). Тем самым она не определена однозначно. Подставляя функцию Карлемана $\widehat{C}_{N}(x, y ; \tau)$ в $(2.2)$, получаем функцию Карлемана $\mathscr{L}_{\tau \mapsto t}^{-1} \widehat{C}_{N}(x, y ; \tau)$ задачи (2.1). При этом возникает вопрос, принадлежит ли семейство ядер $\widehat{C}_{N}(x, y ; \tau)$ множеству значений преобразования Лапласа. В [17] для специального класса областей $\mathscr{X}$ из $\mathbb{R}^{n}$ он сводится к такому же вопросу для обратного преобразования Лапласа целой функции $\cos \sqrt{\tau}$ на плоскости комплексной переменной $\tau$. В следующем разделе представим теоретические обоснования, которые позволят определить $\mathscr{L}_{\tau \mapsto t}^{-1} \cos \sqrt{\tau}$ как аналитический функционал с носителем в $t=0$. Мы не предпринимали попыток развить общую теорию, поскольку наших вычислений достаточно для прикладных целей.

\section{3. Преобразование Лапласа}

Для интегрируемой функции $u$ от $t \in[0, \infty)$ определим преобразование Лапласа по формуле

$$
\mathscr{L} u(\tau)=\int_{0}^{\infty} e^{-\imath \tau t} u(t) d t
$$

для всех $\tau \in \mathbb{R}$, что является преобразованием Фурье функции класса $L^{1}(\mathbb{R})$, бесконечно малой при $t<0$ и совпадающей с $u(t)$ при $t \geq 0$. На самом деле $\mathscr{L} u$ может быть продолжено до аналитической функции комплексной переменной $\tau=\varrho+\imath \sigma$ в нижней полуплоскости $\sigma \leq 0$, поскольку

$$
\mathscr{L} u(\tau)=\int_{0}^{\infty} e^{-\imath \varrho t}\left(e^{\sigma t} u(t)\right) d t=\mathscr{L}\left(e^{\sigma t} u\right)(\varrho)
$$

для всех $\varrho \in \mathbb{R}$. Если ограничиться сужением на горизонтальную линию $\operatorname{Re} \tau=$ $\gamma$ в нижней полуплоскости, то преобразование Лапласа будет корректно определено для всех функций $u$ на полуоси, удовлетворяющих условию $e^{\gamma t} u \in L^{1}[0, \infty)$, а обратное преобразование Лапласа будет задано формулой

$$
\mathscr{L}^{-1} f(t)=\frac{1}{2 \pi} \int_{\operatorname{Im} \tau=\gamma} e^{\imath t \tau} f(\tau) d \tau
$$


для всех $t \in[0, \infty)$. Интеграл в правой части (3.1) понимается как обратное преобразование Фурье распределения умеренного роста на $\mathbb{R}$.

Если $e^{\gamma t} u \in L^{1}[0, \infty)$, то преобразование Лапласа $u$ является аналитической функцией на полуплоскости $\operatorname{Im} \tau<\gamma$ и

$$
|\mathscr{L} u(\tau)|=\left|\int_{0}^{\infty} e^{-\imath \varrho t}\left(e^{\sigma t} u(t)\right) d t\right| \leq\left\|e^{(\operatorname{Im} \tau) t} u\right\|_{L^{1}[0, \infty)}
$$

при условии, что $\operatorname{Im} \tau \leq \gamma$. С другой стороны,

$$
\cos \sqrt{\tau}=\frac{1}{2}\left(e^{\imath \sqrt{\tau}}+e^{-\imath \sqrt{\tau}}\right)=\frac{1}{2}\left(e^{\sqrt{r}(-\sin (\varphi / 2)+\imath \cos (\varphi / 2))}+e^{\sqrt{r}(\sin (\varphi / 2)-\imath \cos (\varphi / 2))}\right),
$$

где $r=|\tau|$ и $\varphi$ - аргумент $\tau$. Если $\tau$ принадлежит нижней полуплоскости $\operatorname{Im} \tau<0$, то можно выбрать аргумент $\tau$ из отрезка $[\pi, 2 \pi]$, тем самым $\varphi / 2 \in$ $[\pi / 2, \pi]$. Следовательно, функция $\sin (\varphi / 2)$ убывает от 1 до 0 при $\varphi \in[\pi, 2 \pi]$, откуда получаем, что для больших $|\tau|$

$$
|\cos \sqrt{\tau}|=\frac{1}{2} \exp \left(\sqrt{|\tau|} \sin \frac{1}{2} \arg \tau\right)+o(1),
$$

т. е. не ограничено при $|\tau| \rightarrow \infty$.

Тем самым целая функция $\cos \sqrt{\tau}$ не может быть преобразованием Лапласа функции $u$ на $[0, \infty)$ такой, что $e^{\gamma t} u \in L^{1}[0, \infty)$ для некоторого $\gamma \leq 0$. Следовательно, обратное преобразование Лапласа не может быть получено в рамках классического операционного анализа (см. [5]). Далее мы расширим область определения $\mathscr{L}$ (ср. [6]).

\section{4. Распределения на полуоси}

Полуось $[0, \infty)$ - замкнутое подмножество $\mathbb{R}$, а значит, есть два естественных способа ввести распределения на $[0, \infty)$. Первый - рассмотреть подпространство $\mathscr{D}^{\prime}(\mathbb{R})$, состоящее из распределений на всем $\mathbb{R}$ с носителем на $[0, \infty)$. Обозначим через $\mathscr{D}_{[0, \infty)}^{\prime}(\mathbb{R})$ это подпространство, снабженное топологией, порожденной $\mathscr{D}^{\prime}(\mathbb{R})$.

Другой подход состоит в рассмотрении непрерывных линейных функционалов на топологическом векторном пространстве $\mathscr{D}[0, \infty)$. Это пространство определено как фактор-пространство $\mathscr{D}(\mathbb{R})$ по замыканию подпространства $\mathscr{D}_{(-\infty, 0)}(\mathbb{R})$, состоящего из всех функций класса $C^{\infty}$ с компактным носителем на $(-\infty, 0)$. Пространство $\mathscr{D}[0, \infty)$ понимается как пространство сужений функций $u \in \mathscr{D}(\mathbb{R})$ на $[0, \infty)$. Через $\mathscr{D}^{\prime}[0, \infty)$ обозначим сопряженное пространство.

Теорема 4.1. Определенные выше пространства $\mathscr{D}_{[0, \infty)}^{\prime}(\mathbb{R})$ и $\mathscr{D}^{\prime}[0, \infty)$ топологически изоморфны.

ДоказАтельство. Пусть $f \in \mathscr{D}_{[0, \infty)}^{\prime}(\mathbb{R})$, т. е. $f \in \mathscr{D}^{\prime}(\mathbb{R})$ и носитель $f$ принадлежит $[0, \infty)$. Если $u \in \mathscr{D}[0, \infty)$, то существует функция $\tilde{u} \in \mathscr{D}(\mathbb{R})$ такая, что $\tilde{u}=u$ на $[0, \infty)$. Положим

$$
\langle f, u\rangle:=\langle f, \tilde{u}\rangle .
$$

Пусть $\tilde{u}$ и $\tilde{\tilde{u}}$ - две функции из $\mathscr{D}(\mathbb{R})$, совпадающие с $u$ на $[0, \infty)$. Тогда $u=\tilde{u}-\tilde{\tilde{u}}$ принадлежит $\mathscr{D}(\mathbb{R})$ и ее носитель лежит на $(-\infty, 0]$. По теореме о спектральном 
синтезе для гладких функций (см. теорему 1.2 .10 в [17]) существует последовательность $\left(u_{k}\right)_{k=1}^{\infty}$ в $\mathscr{D}(\mathbb{R})$ такая, что для любой $u_{k}$ носитель лежит в $(-\infty, 0)$ и $u_{k} \rightarrow u$ в топологии $\mathscr{D}(\mathbb{R})$. Тем самым

$$
\langle f, \tilde{u}\rangle=\langle f, \tilde{\tilde{u}}\rangle+\langle f, \tilde{u}-\tilde{\tilde{u}}\rangle=\langle f, \tilde{\tilde{u}}\rangle+\lim _{k \rightarrow \infty}\left\langle f, u_{k}\right\rangle=\langle f, \tilde{\tilde{u}}\rangle,
$$

т. е. $\langle f, u\rangle$ не зависит от выбора продолжения $u$ на все множество $\mathbb{R}$.

Таким образом построено инъективное отображение $\mathscr{D}_{[0, \infty)}^{\prime}(\mathbb{R}) \hookrightarrow \mathscr{D}^{\prime}[0, \infty)$. Следующей целью будет доказательство его сюръективности. Пусть $f-$ произвольное распределение из $\mathscr{D}^{\prime}[0, \infty)$. Если $u \in \mathscr{D}(\mathbb{R})$, то сужение $u$ на $[0, \infty)$ принадлежит $\mathscr{D}[0, \infty)$. Определим

$$
\langle\tilde{f}, u\rangle=\left\langle f, u \uparrow_{[0, \infty)}\right\rangle .
$$

Ясно, что $\tilde{f} \in \mathscr{D}^{\prime}(\mathbb{R})$. Пусть носитель $u$ не лежит на $[0, \infty)$. Тогда $\langle\tilde{f}, u\rangle=0$ при условии, что носитель $\tilde{f}$ лежит в $[0, \infty)$. Очевидно, что $f$ является образом $\tilde{f}$ при вложении $\mathscr{D}_{[0, \infty)}^{\prime}(\mathbb{R}) \hookrightarrow \mathscr{D}^{\prime}[0, \infty)$.

Явная конструкция изоморфизма $\mathscr{D}_{[0, \infty)}^{\prime}(\mathbb{R}) \cong \mathscr{D}^{\prime}[0, \infty)$ показывает, что он топологический.

По теореме 4.1 преобразование Лапласа распределения $f \in \mathscr{D}^{\prime}[0, \infty)$ просто является преобразованием Фурье $\mathscr{F} f$ этого распределения, определенного в терминах $\mathscr{D}_{[0, \infty)}^{\prime}(\mathbb{R})$. Для произвольного $f \in \mathscr{D}^{\prime}[0, \infty)$ теория развита в [9], а для $f \in \mathscr{D}^{\prime}[0, \infty)$ с компактным носителем преобразование Лапласа задается формулой

$$
\mathscr{L} f(\tau)=\mathscr{F} f(\tau)=\langle f, \exp (-\imath \tau \cdot)\rangle
$$

для всех $\tau \in \mathbb{C}$. Очевидно, что это целая функция от $\tau$. Так как носитель $f$ компактен, найдутся целое $N \geq 0$ и функция $u_{N} \in L^{1}[0, \infty)$ с компактным носителем такие, что $f=\partial^{N} u_{N}$. Тем самым

$$
|\mathscr{L} f(\tau)|=\left|(\imath \tau)^{N} \int_{0}^{\infty} e^{-\imath \varrho t}\left(e^{\sigma t} u_{N}(t)\right) d t\right| \leq\langle\tau\rangle^{N}\left\|e^{(\operatorname{Im} \tau) t} u_{N}\right\|_{L^{1}[0, \infty)}
$$

для всех $\tau$. Еще раз убеждаемся, что $\cos \sqrt{\tau}$ не принадлежит множеству значений преобразования Лапласа на $\mathscr{D}^{\prime}[0, \infty)$.

\section{5. Формальное вычисление обратного преобразования Лапласа от $\cos \sqrt{\tau}$}

Далее представим формальное вычисление обратного преобразования Лапласа целой функции $\cos \sqrt{\tau}$ с помощью формулы (3.1). Эта функция раскладывается в степенной ряд

откуда

$$
\cos \sqrt{\tau}=\sum_{n=0}^{\infty} \frac{(-1)^{n}}{(2 n) !} \tau^{n}
$$

$$
\begin{array}{r}
\mathscr{L}_{\tau \mapsto t}^{-1} \cos \sqrt{\tau}=\frac{1}{2 \pi} \int_{\operatorname{Im} \tau=\gamma} e^{\imath t \tau} \cos \sqrt{\tau} d \tau=\frac{1}{2 \pi} \int_{\operatorname{Im} \tau=\gamma} e^{\imath t \tau} \sum_{n=0}^{\infty} \frac{(-1)^{n}}{(2 n) !} \tau^{n} d \tau \\
=\sum_{n=0}^{\infty} \frac{(-1)^{n}}{(2 n) !} \frac{1}{2 \pi} \int_{\operatorname{Im} \tau=\gamma} e^{\imath t \tau} \tau^{n} d \tau=\sum_{n=0}^{\infty} \frac{(-1)^{n}}{(2 n) !}\left(\frac{1}{\imath} \frac{\partial}{\partial t}\right)^{n} \frac{1}{2 \pi} \int_{\operatorname{Im} \tau=\gamma} e^{\imath t \tau} d \tau \\
=\sum_{n=0}^{\infty} \frac{(-1)^{n}}{(2 n) !}\left(\frac{1}{\imath} \frac{\partial}{\partial t}\right)^{n} \delta(t)=\sum_{n=0}^{\infty} \frac{\imath^{n}}{(2 n) !} \delta^{(n)}(t) .
\end{array}
$$


Слагаемые в правой части (5.1) имеют носители в $t=0$. Однако этот ряд не является распределением на $[0, \infty)$. Действительно, для произвольной функции $u(t)$ класса $C^{\infty}$ в окрестности $t=0$ если $u-$ не аналитическая функция в $t=0$, то ряд

$$
\left\langle\sum_{n=0}^{\infty} \frac{\imath^{n}}{(2 n) !} \delta^{(n)}(t), u\right\rangle=\sum_{n=0}^{\infty} \frac{(-\imath)^{n}}{(2 n) !} u^{(n)}(0)
$$

может не быть сходящимся. Следовательно, правая часть в (5.1) должна быть определена в терминах аналитических функционалов.

Обратно, интерпретируя ряд в правой части (5.1) как аналитический функционал, легко получаем

$$
\begin{aligned}
\mathscr{L}_{t \mapsto \tau}\left(\sum_{n=0}^{\infty} \frac{\imath^{n}}{(2 n) !} \delta^{(n)}(t)\right) & =\left\langle\sum_{n=0}^{\infty} \frac{\imath^{n}}{(2 n) !} \delta^{(n)}(t), \exp (-\imath \tau t)\right\rangle \\
= & \sum_{n=0}^{\infty} \frac{\imath^{n}}{(2 n) !}\left\langle\delta^{(n)}(t), \exp (-\imath \tau t)\right\rangle=\sum_{n=0}^{\infty} \frac{\imath^{n}}{(2 n) !}(\imath \tau)^{n}=\cos \sqrt{\tau},
\end{aligned}
$$

что и требовалось.

\section{6. Преобразование Лапласа аналитических функционалов}

Пусть 0 - начало координат в $\mathbb{R}$ и $\mathscr{A}_{0}$ - семейство аналитических функций, определенных в окрестности 0: каждая функция аналитична в своей окрестности. Назовем две функции эквивалентными в 0, если они совпадают в некоторой окрестности 0 . Класс эквивалентности, порождаемый этим отношением, называется ростком аналитических функций в 0. Множество ростков аналитических функций в 0 наследует структуру кольца от аналитических функций, где операции применяются к представителям классов. Для упрощения используем то же обозначение $\mathscr{A}_{0}$ для кольца ростков аналитических функций в 0 .

Любая аналитическая функция $u$ переменной $t$ в окрестности начала координат может быть разложена в степенной ряд

$$
u(t)=\sum_{n=0}^{\infty} c_{n} t^{n}
$$

на некотором интервале $(-r, r)$, где $r>0$. Наибольшее $r$ с этим свойством называется радиусом сходимости ряда и задается формулой Коши - Адамара

$$
r=\frac{1}{\limsup _{n \rightarrow \infty} \sqrt[n]{\left|c_{n}\right|}}
$$

где $c_{n}=\frac{u^{(n)}(0)}{n !}$.

Ряд (6.1) сходится в единичном круге $\mathbb{D}$ с центром в начале координат на комплексной плоскости, причем сходимость равномерна по $t$ на компактных подмножествах $\mathbb{D}$. Следовательно, $u(t)$ однозначно доопределяется до комплексной аналитической (голоморфной) функции в круге $\mathbb{D}$. В силу этого наделим кольцо $\mathscr{A}_{0}$ локально выпуклой топологией, обладающей свойством Гейне Бореля. А именно, последовательность $\left(\left[u_{k}\right]\right)_{k=1}^{\infty}$ ростков в $\mathscr{A}_{0}$ называется сходящейся $\kappa$ ростку $[u] \in \mathscr{A}_{0}$, если существует окрестность $U$ начала координат 
в $\mathbb{C}$ такая, что в любом из классов $\left[u_{k}\right]$ и $[u]$ есть аналитический представитель $u_{k}$ и $u$ в $U$ соответственно, а $\left(u_{k}\right)_{k=1}^{\infty}$ сходится к $u$ равномерно на компактных подмножествах в $U$. Описание топологии стандартно.

Далее приведем результат относительно сходящихся последовательностей, имеющий место в любом бочечном топологическом векторном пространстве. Любое пространство Фреше или индуктивный предел таких пространств является бочечным топологическим векторным пространством. Бочечные топологические векторные пространства важны, поскольку это самые слабые топологические объекты, на которые распространяется теорема Банаха - Штейнгауза; следующее утверждение является простым следствием этого.

Теорема 6.1. Пусть $V$ - бочечное топологическое векторное пространство, а $V^{\prime}$ - сопряженное к нему. Пусть $\left(f_{k}\right)_{k=1}^{\infty}-$ последовательность в $V^{\prime}$, сходящаяся поточечно на $V$, обозначим ее предел через $f$. Тогда $f \in V^{\prime}$. Более того, если $\left(u_{k}\right)_{k=1}^{\infty}-$ последовательность в $V$ с пределом $u$, то

$$
\lim _{k \rightarrow \infty}\left\langle f_{k}, u_{k}\right\rangle=\langle f, u\rangle .
$$

ДокАзАтельство. По теореме Банаха - Штейнгауза $\left(f_{k}\right)_{k=1}^{\infty}$ сходится к $f$ равномерно на компактах и $f$ непрерывна, т. е. $f \in V^{\prime}$. Запишем

$$
\langle f, u\rangle-\left\langle f_{k}, u_{k}\right\rangle=\left\langle f, u-u_{k}\right\rangle+\left\langle f-f_{k}, u_{k}\right\rangle .
$$

В силу непрерывности $f$ выражение в первых скобках стремится к нулю при $k \rightarrow \infty$. То же самое верно и для выражения во вторых скобках, поскольку $\left(u_{k}\right)_{k=1}^{\infty}-$ компактное множество.

Обозначим через $\mathscr{A}_{0}^{\prime}$ пространство, сопряженное к $\mathscr{A}_{0}^{\prime}$. Элементы $\mathscr{A}_{0}^{\prime}$ называются аналитическими функционалами. Теория аналитических функционалов имеет много общего с теорией распределений Шварца (см. [7, гл. 9]). Для произвольной $f \in \mathscr{A}_{0}^{\prime}$ ее преобразование Лапласа

$$
\mathscr{L} f(\tau)=\langle f, \exp (-\imath \tau \cdot)\rangle
$$

является целой функцией переменной $\tau \in \mathbb{C}$, поскольку $\exp (-\imath \tau t)$ - целая функция двух комплексных переменных $(t, \tau) \in \mathbb{C}^{2}$.

Вычисления из разд. 5 будут завершены, если докажем, что формальный ряд

$$
f(t)=\sum_{n=0}^{\infty} \frac{\imath^{n}}{(2 n) !} \delta^{(n)}(t)
$$

сходится в пространстве аналитических функционалов в начале координат. Для этого согласно теореме 6.1 достаточно показать, что последовательность

$$
\left\langle\sum_{n=0}^{N} \frac{\imath^{n}}{(2 n) !} \delta^{(n)}, u\right\rangle=\sum_{n=0}^{N}\left(\frac{1}{\imath}\right)^{n} \frac{n !}{(2 n) !} \frac{u^{(n)}(0)}{n !}
$$

сходится для любой аналитической функции $u$ в окрестности $0 \in \mathbb{C}$. Для произвольной $u \in \mathscr{A}_{0}$ имеем

$$
\limsup _{n \rightarrow \infty} \sqrt[n]{\left|\frac{u^{(n)}(0)}{n !}\right|}<\infty
$$


С другой стороны, по формуле Стирлинга получаем

$$
\frac{n !}{(2 n) !} \sim \frac{n^{n} e^{-n} \sqrt{2 \pi n}}{(2 n)^{2 n} e^{-2 n} \sqrt{2 \pi 2 n}}=\frac{1}{\sqrt{2}} \frac{1}{\left(4 \frac{n}{e}\right)^{n}} \rightarrow 0
$$

при $n \rightarrow \infty$. Тем самым формальный ряд $f(t)$ сходится в пространстве $\mathscr{A}_{0}^{\prime}$, что и требовалось.

Техники, развитые нами в ходе вычислений обратного преобразования Лапласа функции $\cos \sqrt{\tau}$, по всей видимости, представляют интерес сами по себе, так как позволяют свести изучение нехарактеристической задачи Коши для параболических уравнений к таким же задачам для эллиптических уравнений при условии, что функции Карлемана последних построены в терминах аналитических функций. Этот подход лежит в основе нескольких методов из [17].

\section{7. Формулы Карлемана для параболических уравнений}

Задача Коши для решения эллиптических уравнений с данными на непустом открытом участке границы хорошо изучена (см. [17]). Известно, что задача $(2.2)$ допускает решение на подпространстве данных $\hat{f}(\cdot, \tau)$ и $\hat{u}_{0}(\cdot, \tau)$ бесконечной коразмерности и приближенное решение этой задачи может быть получено по так называемой формуле Карлемана

$$
\hat{u}(x, \tau)=\lim _{N \rightarrow \infty}\left(-\int_{S} G_{A}\left(\widehat{C}_{N}(x, \cdot ; \tau), t(\hat{u})\right)+\int_{\mathscr{X}} \widehat{C}_{N}(x, \cdot ; \tau)(A-\imath \tau) \hat{u} d y\right)
$$

для всех $x \in \mathscr{X}$, где $G_{A}(g, u)$ - бидифференциальный оператор порядка, на единицу меньшего порядка $A$.

Будем считать, что обратное преобразование Лапласа возможно при переходе к пределу в последней формуле. По известной теореме о свертке для преобразования Лапласа имеем

$$
\begin{aligned}
\mathscr{L}^{-1} G_{A}\left(\widehat{C}_{N}(x, \cdot ; \tau), t(\hat{u})\right)=\mathscr{L}^{-1} G_{A} & \left(\mathscr{L} \mathscr{L}^{-1} \widehat{C}_{N}(x, \cdot ; \tau), \mathscr{L} t(u)\right) \\
= & \int_{0}^{t} G_{A}\left(\left(\mathscr{L}^{-1} \widehat{C}_{N}\right)\left(x, \cdot ; t-t^{\prime}\right), t(u)\left(\cdot, t^{\prime}\right)\right) d t^{\prime} .
\end{aligned}
$$

Аналогично

$$
\begin{aligned}
\mathscr{L}^{-1}\left(\widehat{C}_{N}(x, \cdot ; \tau)(A-\imath \tau) \hat{u}\right) & \\
= & \int_{0}^{t}\left(\mathscr{L}^{-1} \widehat{C}_{N}\right)\left(x, \cdot ; t-t^{\prime}\right)\left(A-\partial_{t^{\prime}}\right) u\left(\cdot, t^{\prime}\right) d t^{\prime}-\left(\mathscr{L}^{-1} \widehat{C}_{N}\right)(x, \cdot ; t) u(\cdot, 0) .
\end{aligned}
$$

Тем самым, применяя обратное преобразование Лапласа к обеим частям равен- 
ства (7.1), приходим к формуле

$$
\begin{aligned}
u(x, t)=\lim _{N \rightarrow \infty}\left(-\int_{\mathscr{X}}\left(\mathscr{L}^{-1} \widehat{C}_{N}\right)(x, \cdot ; t) u(\cdot, 0) d y\right. \\
\quad-\int_{S} \int_{0}^{t} G_{A}\left(\left(\mathscr{L}^{-1} \widehat{C}_{N}\right)\left(x, \cdot ; t-t^{\prime}\right), t(u)\right) d t^{\prime} \\
\left.\quad+\int_{\mathscr{X}} \int_{0}^{t}\left(\mathscr{L}^{-1} \widehat{C}_{N}\right)\left(x, \cdot ; t-t^{\prime}\right)\left(A-\partial_{t^{\prime}}\right) u d y d t^{\prime}\right)
\end{aligned}
$$

для всех $(x, t) \in \mathscr{C}$.

Положим

$$
C_{N}(x, y ; t)=\mathscr{L}^{-1} \widehat{C}_{N}(x, y ; \tau)=\frac{1}{2 \pi} \int_{\operatorname{Im} \tau=\gamma} e^{\imath t \tau} \widehat{C}_{N}(x, y ; \tau) d \tau
$$

для $(x, y)$ вне диагонали в $\mathscr{X} \times \overline{\mathscr{X}}$ и при $t>0$, где $\gamma-$ достаточно малое отрицательное число. Прямые вычисления показывают, что

$$
\left(A(y, D)-\partial_{t}\right) C_{N}(x, y ; t)=\frac{1}{2 \pi} \int_{\operatorname{Im} \tau=\gamma} e^{\imath t \tau}(A(y, D)-\imath \tau) \widehat{C}_{N}(x, y ; \tau) d \tau=0
$$

для $(x, y)$ и $t$ в области определения $C_{N}$. Более того, $C_{N}(x, y ; t)$ в некотором смысле стремится к нулю по $y$ вне $S$ на границе $\mathscr{X}$ при $N \rightarrow \infty$. Тем самым последовательность ядер $C_{N}(x, y ; t)$ является обобщением для задачи $(2.1)$ понятия функции Карлемана в задаче Коши для эллиптических уравнений, развитое в [17]. Если функция Карлемана $C_{N}$ задана явно, то легко получить формулу для приближенного решения (2.1).

Теорема 7.1. Если $C_{N}(x, y ; t)$ - функция Карлемана задачи $(2.1)$, то формула

$$
u_{N}=\mathscr{P}_{i, N}(u(\cdot, 0))+\mathscr{P}_{l s, N}\left(u_{0}\right)+\mathscr{P}_{v, N}(f)
$$

дает приближенное решение задачи в цилиндре $\mathscr{C}$, где

$$
\begin{gathered}
\mathscr{P}_{i, N}(u(\cdot, 0))(x, t)=-\int_{\mathscr{X}} C_{N}(x, y ; t) u(y, 0) d y \\
\mathscr{P}_{l s, N}\left(u_{0}\right)(x, t)=-\int_{S} \int_{0}^{t} G_{A}\left(C_{N}\left(x, y ; t-t^{\prime}\right), u_{0}\left(y, t^{\prime}\right)\right) d t^{\prime}, \\
\mathscr{P}_{v, N}(f)(x, t)=-\int_{\mathscr{X}} \int_{0}^{t} C_{N}\left(x, y ; t-t^{\prime}\right) f\left(y, t^{\prime}\right) d y d t^{\prime} .
\end{gathered}
$$

Заметим, что значения $u(x, 0)$ на нижнем основании цилиндра однозначно определены значениями $u(x, t)$ для малых $t>0$, если $u$ непрерывна в некотором смысле вплоть до $t=0$. Таким образом, $u(x, 0)$ определяются однозначно по значениям $t(u)$ в полосе $S \times(0, \infty)$. Однако формулы Карлемана наиболее просты, если они включают начальные значения $u(x, 0)$.

Явные формулы Карлемана для решения задачи Коши для уравнения теплопроводности в определенных областях $\mathscr{X} n$-мерного евклидова пространства можно найти в $[16,18]$. 


\section{ЛИТЕРАТУРА}

1. Bochner S. Vorlesungen über Fouriersche Integrale // Mathematik und ihre Anwendungen. Bd. 12. Leipzig: Akad. Verlagsges., 1932.

2. Carleman T. L'intégrale de Fourier et questions qui s'y rattachent. Uppsala: Almqvist \& Wiksells, 1944 (Publ. Sci. Inst. Mittag-Leffler; V. 1).

3. Schwartz L. Théorie des distributions. Paris: Hermann, 1950-1951.

4. Petrowsky I. G. Über das Cauchysche Problem für ein System linearer partieller Differentialgleichungen im Gebiete der nichtanalytischen Funktionen // Bull. Univ. Etat. Moscou, Ser. Int., Sect. A, Math. et Mecan. 1938. V. 1, N 7. P. 1-74.

5. Paley R., Wiener N. Fourier transforms in the complex domain. Providence, RI: Amer. Math. Soc., 1934. (Colloquium Publ.; V. 19).

6. Schwartz L. Transformation de Laplace des distributions // Comm. Sém. Math. Univ. Lund, Tome suppl. 1952. P. 196-206.

7. Hörmander L. The analysis of linear partial differential operators. I. Distribution theory and Fourier analysis. Berlin: Springer-Verl., 1983.

8. Гельфанд И. М., Шилов Г. Е. Преобразования Фурье быстро растущих функций и вопросы единственности решения задачи Коши // Успехи мат. наук. 1953. Т. 8, № 6. С. 3-54.

9. Ehrenpreis L. Analytic functions and the Fourier transform of distributions, I // Ann. Math. 1956. V. 63. P. 129-159.

10. Shambayati R., Zielezny Z. On Fourier transforms of distributions with one-sided support // Proc. Amer. Math. Soc. 1983. V. 88. P. 237-243.

11. Cheikh Y. Ben. Generalised Paley-Wiener theorem and generalized convolution associated with the differential operator $\partial_{z}^{n}$ in the complex domain // Integral Transforms Spec. Funct. 2000. V. 9. P. 245-256.

12. Carlsson M., Wittsten J. A note on holomorphic functions and the Fourier-Laplace transform // Math. Scand. 2017. V. 120, N 2. P. 225-242.

13. Sebastião e Silva J. Les fonctions analytiques comme ultra-distributions dans le calcul opérationnel // Math. Ann. 1958. V. 136. P. 58-96.

14. Yoshinaga $K$. On spaces of distributions of exponential growth // Bull. Kyushu Inst. Tech. Math. Nat. Sci. 1960. V. 6. P. 1-60.

15. Chung J., Chung S.-Y., Kim D. Characterizations of the Gelfand-Shilov spaces via Fourier transforms // Proc. Amer. Math. Soc. 1996. V. 124, N 7. P. 2101-2108.

16. Махмудов K. О., Махмудов О. И., Тарханов Н. Н. Нестандартная задача Коши для уравнения теплопроводности // Мат. заметки. 2017. Т. 102, № 2. С. 270-283.

17. Tarkhanov N. The Cauchy problem for solutions of elliptic equations. Berlin: Akad.-Verl., 1995.

18. Kurilenko I. A., Shlapunov A. A. On Carleman-type formulas for solutions to the heat equation // J. Sib. Fed. Univ., Math. Phys. 2019. V. 12, N 4. P. 421-433.

Поступила в редакцию 11 ноября 2019 г.

После доработки 28 апреля 2020 г.

Принята к публикации 17 июня 2020 г.

Wafaa Chelkh (Челк Вафаa)

Institute of Mathematics, University of Potsdam, Karl-Liebknecht-Str. 24/25, 14476 Potsdam, Germany

chelkh@uni-potsdam.de

Ibrahim Ly (Ли Ибрахим)

Départment de Mathématique, Université Ouaga 1, Pr. JKZ 03, B.P. 7021 Ouaga 03, Burkina Faso

lyibrahim@gmx.de

Тарханов Николай Николаевич

Institute of Mathematics, University of Potsdam,

Karl-Liebknecht-Str. 24/25, 14476 Potsdam, Germany

tarkhanov@math.uni-potsdam.de 